\title{
On Stress-Strength Reliability with a Time-Dependent Strength
}

\author{
Serkan Eryilmaz \\ Department of Industrial Engineering, Atilim University, Incek, 06836 Ankara, Turkey \\ Correspondence should be addressed to Serkan Eryilmaz; seryilmaz@atilim.edu.tr
}

Received 25 July 2012; Accepted 21 December 2012

Academic Editor: Shey-Huei Sheu

Copyright (c) 2013 Serkan Eryilmaz. This is an open access article distributed under the Creative Commons Attribution License, which permits unrestricted use, distribution, and reproduction in any medium, provided the original work is properly cited.

\begin{abstract}
The study of stress-strength reliability in a time-dependent context needs to model at least one of the stress or strength quantities as dynamic. We study the stress-strength reliability for the case in which the strength of the system is decreasing in time and the stress remains fixed over time; that is, the strength of the system is modeled as a stochastic process and the stress is considered to be a usual random variable. We present stochastic ordering results among the lifetimes of the systems which have the same strength but are subjected to different stresses. Multicomponent form of the aforementioned stress-strength interference is also considered. We illustrate the results for the special case when the strength is modeled by a Weibull process.
\end{abstract}

\section{Introduction}

Stress-strength models are of special importance in reliability literature and engineering applications. A technical system or unit may be subjected to randomly occurring environmental stresses such as pressure, temperature, and humidity and the survival of the system heavily depends on its resistance. In the simplest form of the stress-strength model, a failure occurs when the strength (or resistance) of the unit drops below the stress. In this case the reliability $R$ is defined as the probability that the unit's strength is greater than the stress, that is, $R=$ $P\{X<Y\}$, where $Y$ is the random strength of the unit and $X$ is the random stress placed on it. This reliability has been widely studied under various distributional assumptions on $X$ and $Y$. (See, e.g., Johnson [1] and Kotz et al. [2] for an extensive and lucid review of the topic.)

In the aforementioned simplest form, stress and strength quantities are considered to be both static. Dynamic modeling of stress-strength interference might offer more realistic applications to real-life reliability studies than static modeling and it enables us to investigate the time-dependent (dynamic) reliability properties of the system. Let $X(t)$ and $Y(t)$ denote the stress that the system is experiencing and strength of the system at time $t$, respectively. Then the lifetime of the system is represented as

$$
T=\inf \{s \geq 0: X(s)>Y(s)\}
$$

The most important characteristic in reliability analysis is the reliability function of a system which is defined as the probability of surviving at time $t$, that is,

$$
R(t)=P\{T>t\} .
$$

This function is also known as the survival function in the reliability literature and its exact formulation is of special importance in engineering applications.

The reliability function for the lifetime random variable given in (1) is

$$
R(t)=P\{X(s)<Y(s), 0 \leq s \leq t\} .
$$

The function given by (3) has been investigated in several papers. Whitmore [3] computed the function $R(t)$ under the assumptions that $X(t)$ and $Y(t)$ are independent Brownian motions. Ebrahimi [4] studied the properties of $R(t)$ assuming the strength of the system $Y(t)$ is decreasing in time.

In this paper, we study $R(t)$ assuming (i) $Y(t)$ is decreasing in time, that is, $P\left\{Y\left(t_{2}\right) \leq Y\left(t_{1}\right)\right\}=1$ for all $t_{1}<t_{2}$ and (ii) $X(t)=X$; that is, stress remains fixed over time (static). The first assumption is common in reality because the system's strength could degrade due to aging. In Section 2, we provide some stochastic ordering results among the lifetimes of the systems which have the same strength but are subjected to different stresses. In Section 3, stress-strength interference is 
considered for multicomponent systems. Finally, in Section 4 some results are presented for the special case when the strength is modeled by a Weibull process.

\section{Reliability and Ordering Properties}

Under the assumptions (i), (ii) $X$ and $Y(t)$ are independent and the reliability function can be formulated as

$$
\begin{aligned}
R(t) & =P\{X<Y(t)\}=\int_{-\infty}^{\infty} F(x) d G_{t}(x) \\
& =\int_{-\infty}^{\infty} \bar{G}_{t}(x) d F(x)
\end{aligned}
$$

where $F(x)=P\{X \leq x\}, G_{t}(x)=P\{Y(t) \leq x\}$ and $\bar{G}_{t}(x)=$ $1-G_{t}(x)$.

The following example illustrates the computation of reliability function for the stochastic strength process given with its analytical form. That is, the strength aging deterioration process is expressed as a function of time, and a random variable.

Example 1. Let $Y(t)$ be defined by

$$
Y(t)=e^{-C \cdot t}, \quad t \geq 0
$$

where $C$ follows Pareto distribution with c.d.f. $F_{C}(x)=1-$ $(\mu / x)^{2}, x>\mu, \mu>0$. Then the c.d.f. of $Y(t)$ is

$$
G_{t}(x)=\frac{\mu^{2} t^{2}}{(\ln x)^{2}}, \quad 0<x<e^{-\mu t} .
$$

Let $X$ have a c.d.f. $F(x)=x^{\theta}, 0<x<1, \theta>0$. Then using (4) we have

$$
\begin{aligned}
R(t) & =\int_{0}^{e^{-\mu t}}\left(1-\frac{\mu^{2} t^{2}}{(\ln x)^{2}}\right) \theta x^{\theta-1} d x \\
& =e^{-\mu t \theta}-t^{2} \mu^{2} \theta \int_{0}^{e^{-\mu t}} \frac{x^{\theta-1}}{(\ln x)^{2}} d x, \quad t \geq 0 .
\end{aligned}
$$

Differentiating $1-R(t)$ w.r.t. $t$ using the rule of differentiation under the integral sign, the p.d.f. of $T$ is found as

$$
f(t)=2 \mu^{2} \theta t \int_{0}^{e^{-\mu t}} \frac{x^{\theta-1}}{(\ln x)^{2}} d x .
$$

Using (8) in (7) we obtain

$$
R(t)=e^{-\mu t \theta}-\frac{1}{2} t f(t) .
$$
get

Integrating both sides of the last equation over $(0, \infty)$ we

$$
E(T)=\frac{2}{3 \mu \theta}
$$

which is the MTTF of the system.
The process defined by (5) can be considered in a more general form given by

$$
Y(t)=e^{-C \cdot S(t)}, \quad t \geq 0,
$$

where $S(0)=0$ and $S(t)$ is a nondecreasing function. In this case the reliability function and MTTF of the system are found to be

$$
\begin{gathered}
R(t)=e^{-\mu \theta S(t)}-S^{2}(t) \mu^{2} \theta \int_{0}^{e^{-\mu S(t)}} \frac{x^{\theta-1}}{(\ln x)^{2}} d x, \\
\operatorname{MTTF}=\int_{0}^{\infty} e^{-\mu \theta S(t)} d t-\frac{1}{2} E\left[\frac{S(T)}{S^{\prime}(T)}\right] .
\end{gathered}
$$

For the system defined in Example 1 it can be easily seen that an increase in $\theta$ leads to a decrease in MTTF of the system. Since $E X=\theta /(\theta+1)$, the larger $\theta$ the harsher the stress and hence the smaller the reliability. This can be theoretically established using the concept of stochastic ordering as in the following lines.

We investigate the behaviour of the lifetime of the system under different stresses in terms of stochastic ordering. In this context, we consider the following stochastic orderings between the lifetimes. Let $X$ and $Y$ be two random variables having cumulative distributions $F$ and $G$, densities $f$ and $g$, hazard rates $r_{F}$ and $r_{G}$, and reversed hazard rates $h_{F}$ and $h_{G}$, respectively. Note that the hazard and reversed hazard rates are defined, respectively, as

$$
r_{F}(t)=\frac{f(t)}{\bar{F}(t)}, \quad h_{F}(t)=\frac{f(t)}{F(t)} .
$$

Usual Stochastic Ordering. $X$ is said to be smaller than $Y$ in usual stochastic ordering if $P\{X>x\} \leq P\{Y>x\}$ for all $x$, or equivalently $E[\Psi(X)] \leq E[\Psi(Y)]$ for all increasing functions $\Psi$ for which the expectations exist. This relation is denoted by $X \stackrel{\text { st }}{\leq} Y$.

Hazard Rate Ordering. $X$ is said to be smaller than $Y$ in hazard rate ordering if $r_{F}(x) \geq r_{G}(x)$ for all $x$ or $\bar{G}(x) / \bar{F}(x)$ is a nondecreasing function of $x$ and we write $X \leq Y$ hr

Reversed Hazard Rate Ordering. $X$ is said to be smaller than $Y$ in the reversed hazard rate order if $h_{F}(x) \leq h_{G}(x)$ for all $x$ or $G(x) / F(x)$ is a nondecreasing function of $x$ and we write $X \stackrel{\text { rh }}{\leq} Y$.

Likelihood Ratio Ordering. $X$ is said to be smaller than $Y$ in likelihood ratio ordering (written as $X \stackrel{\operatorname{lr}}{\leq} Y$ ) if $g(x) / f(x)$ is nondecreasing function of $x$.

We have the following implications among these orderings:

$$
X \stackrel{\operatorname{lr}}{\leq} Y \Longrightarrow\left(\begin{array}{c}
X \stackrel{\text { hr }}{\leq} Y \\
X \stackrel{\text { rh }}{\leq} Y
\end{array}\right) \Longrightarrow X \stackrel{\text { st }}{\leq} Y .
$$

For more details on stochastic orderings refer to Shaked and Shanthikumar [5].

The following concepts will be useful for the next section. 
Definition 2. A function $k(x, y)$ is said to be sign regular of order $2\left(\mathrm{SR}_{2}\right)$ if $\varepsilon_{1} k(x, y) \geq 0$ and

$$
\varepsilon_{2}\left|\begin{array}{ll}
k\left(x_{1}, y_{1}\right) & k\left(x_{1}, y_{2}\right) \\
k\left(x_{2}, y_{1}\right) & k\left(x_{2}, y_{2}\right)
\end{array}\right| \geq 0
$$

whenever $x_{1}<x_{2}, y_{1}<y_{2}$, and $\varepsilon_{i} \in\{-1,1\}$ for $i=1,2$.

If the conditions given in Definition 2 hold with $\varepsilon_{1}=1$ and $\varepsilon_{2}=1$ then $k$ is said to be totally positive of order $2\left(\mathrm{TP}_{2}\right)$; and $k$ is said to be reverse regular of order $2\left(\mathrm{RR}_{2}\right)$ if they hold with $\varepsilon_{1}=1$ and $\varepsilon_{2}=-1$.

Proposition 3. Let $T_{i}$ denote the lifetime of the system whose stress-strength pair is $\left(X_{i}, Y(t)\right), i=1,2$. Then

(a) if $X_{1} \stackrel{\text { st }}{\leq} X_{2}$ then $T_{1} \stackrel{\text { st }}{\geq} T_{2}$;

(b) if $X_{1} \stackrel{\text { rh }}{\leq} X_{2}$ and $g(x, t)=(\partial / \partial x) G_{t}(x)$ is $\mathrm{RR}_{2}$ in $(x, t)$ then $T_{1} \stackrel{\text { hr }}{\geq} T_{2}$;

(c) if $X_{1} \stackrel{\operatorname{lr}}{\leq} X_{2}$ and $h(x, t)=(\partial / \partial t) G_{t}(x)$ is $\mathrm{RR}_{2}$ in $(x, t)$ then $T_{1} \stackrel{\operatorname{lr}}{\geq} T_{2}$.

Proof. The proof of (a) immediately follows because $P\left\{T_{i} \leq\right.$ $t\}=E\left[G_{t}\left(X_{i}\right)\right], i=1,2$, and $G_{t}(x)$ is increasing in $x$. The proofs of (b) and (c) can be obtained as an application of Theorems 1.B.14, 1.B.52, and 1.C.17 in Shaked and Shanthikumar [5]. These results are obtained using basic composition formula of Karlin [6].

Example 4. Consider the process defined in Example 1 with $F_{C}(x)=1-e^{-x}, x>0$ and let $X_{i}$ have a c.d.f. $F_{i}(x)=x^{\theta_{i}}$, $0<x<1, i=1,2$. In this case

$$
\begin{gathered}
G_{t}(x)=x^{1 / t}, \quad 0<x<1, \\
h(x, t)=\frac{\partial}{\partial t} G_{t}(x)=-\frac{1}{t^{2}} x^{1 / t} \ln x, \\
g(x, t)=\frac{\partial}{\partial x} G_{t}(x)=\frac{1}{t} x^{1 / t-1} .
\end{gathered}
$$

For any $x_{1}<x_{2}$ and $t_{1}<t_{2}$

$$
\begin{aligned}
& h\left(x_{1}, t_{1}\right) h\left(x_{2}, t_{2}\right)-h\left(x_{1}, t_{2}\right) h\left(x_{2}, t_{1}\right) \\
& \quad=\frac{1}{t_{1}^{2} t_{2}^{2}} \ln x_{1} \ln x_{2}\left[x_{1}^{1 / t_{1}} x_{2}^{1 / t_{2}}-x_{2}^{1 / t_{1}} x_{1}^{1 / t_{2}}\right] \leq 0
\end{aligned}
$$

which implies the $\mathrm{RR}_{2}$ property of $h(x, t)$. If $\theta_{1} \leq \theta_{2}$ then $X_{1} \stackrel{\operatorname{lr}}{\leq} X_{2}$. Thus by the Proposition 3 we have $T_{1} \stackrel{\operatorname{lr}}{\geq} T_{2}$. Similarly, $g(x, t)$ is also $\mathrm{RR}_{2}$ in $(x, t)$. If $\theta_{1} \leq \theta_{2}$ then $X_{1} \stackrel{\text { rh }}{\leq} X_{2}$ and hence by the Proposition 3 we have $T_{1} \stackrel{\text { hr }}{\geq} T_{2}$.

\section{Multicomponent Setup}

In the previous sections we analyzed stress-strength reliability for a single component system. Most of the engineering systems consist of several components and the components might have different statistical properties. Multicomponent stress-strength reliability in a static form has been studied in various papers including Bhattacharyya and Johnson [7], Chandra and Owen [8], Johnson [1], Pandey et al. [9], Eryilmaz [10], and Eryilmaz [11].

Assume that a system consists of $n$ components and the deteriorating strength of the $i$ th component at time $t$ is denoted by the process $Y_{i}(t), i=1,2, \ldots, n$. The components are subjected to a common random stress $X$. If $T_{i}$ denotes the lifetime of the $i$ th component then the joint survival function of $T_{1}, T_{2}, \ldots, T_{n}$ is given by

$$
\begin{aligned}
& R\left(t_{1}, t_{2}, \ldots, t_{n}\right) \\
& \quad=P\left\{T_{1}>t_{1}, T_{2}>t_{2}, \ldots, T_{n}>t_{n}\right\} \\
& \quad=P\left\{X<Y_{1}\left(t_{1}\right), X<Y_{2}\left(t_{2}\right), \ldots, X<Y_{n}\left(t_{n}\right)\right\} .
\end{aligned}
$$

If the components are independent then we have

$$
R\left(t_{1}, t_{2}, \ldots, t_{n}\right)=\int \prod_{i=1}^{n} P\left\{Y_{i}\left(t_{i}\right)>x\right\} d F(x)
$$

From (19) it follows that the lifetimes of the components are dependent even if the strengths of them are independent. This positive dependence among the lifetimes arises from common environmental stress characterized by $X$. There are many types of positive dependence. The likelihood ratio (or $\mathrm{TP}_{2}$ ) dependence as the strongest notion of positive dependence is defined as follows. Let $T_{1}, T_{2}$ have the joint probability density $f\left(t_{1}, t_{2}\right)$. Then recall from Definition 2 that $f\left(t_{1}, t_{2}\right)$ is $\mathrm{TP}_{2}$ if

$$
\left|\begin{array}{ll}
f\left(t_{1}^{(1)}, t_{2}^{(1)}\right) & f\left(t_{1}^{(1)}, t_{2}^{(2)}\right) \\
f\left(t_{1}^{(2)}, t_{2}^{(1)}\right) & f\left(t_{1}^{(2)}, t_{2}^{(2)}\right)
\end{array}\right| \geq 0
$$

for $t_{1}^{(1)}<t_{1}^{(2)}$ and $t_{2}^{(1)}<t_{2}^{(2)}$. The random variables $T_{1}$ and $T_{2}$ are said to be likelihood ratio (or $\mathrm{TP}_{2}$ ) dependent if their joint density is $\mathrm{TP}_{2}$.

The following result can be proved using the basic composition formula of Karlin [6] together with

$$
f\left(t_{1}, t_{2}\right)=\int h^{(1)}\left(x, t_{1}\right) h^{(2)}\left(x, t_{2}\right) d F(x),
$$

where $h^{(i)}(x, t)=(\partial / \partial t) G_{t, i}(x)$ and $G_{t, i}(x)=P\left\{Y_{i}(t) \leq x\right\}$, $i=1,2$.

Proposition 5. If $h^{(1)}\left(x, t_{1}\right)$ is $T P_{2}\left(R R_{2}\right)$ in $\left(t_{1}, x\right)$ and $h^{(2)}\left(x, t_{2}\right)$ is $T P_{2}\left(R R_{2}\right)$ in $\left(x, t_{2}\right)$, then $T_{1}$ and $T_{2}$ are likelihood ratio dependent.

Example 6. Let $Y_{i}(t)=e^{-C_{i} t}, t \geq 0, i=1,2$ and $C_{i}$ be an exponential random variable with c.d.f. $F_{C_{i}}(x)=1-e^{-\mu_{i} x}$, $x>0$. Also assume that the common random stress $X$ has 
c.d.f. $F(x)=x^{\theta}, 0<x<1$. In this case the joint survival function of $T_{1}$ and $T_{2}$ is found to be

$$
\begin{aligned}
R\left(t_{1}, t_{2}\right)= & 1-\frac{\theta t_{1}}{\mu_{1}+\theta t_{1}}-\frac{\theta t_{2}}{\mu_{2}+\theta t_{2}} \\
& +\frac{\theta}{\mu_{1} / t_{1}+\mu_{2} / t_{2}+\theta}, \quad t_{1}, t_{2} \geq 0 .
\end{aligned}
$$

Since

$$
h^{(i)}(x, t)=-\frac{\mu_{i}}{t^{2}} x^{\mu_{i} / t} \ln x
$$

is $\mathrm{RR}_{2}$ for $i=1,2, T_{1}$ and $T_{2}$ are likelihood ratio dependent.

Consider a system $\phi$ with $n$ components which has two possible states; $\phi=1$ if the system is functioning and $\phi=$ 0 if the system has failed. Since the state of the system is determined by the states of its components we can write $\phi=\phi\left(x_{1}, \ldots, x_{n}\right)$, where $x_{i}=1$ if the $i$ th component is functioning and $x_{i}=0$ if it has failed. The function $\phi$ is called structure function. A system with structure function $\phi$ is coherent if $\phi$ is nondecreasing in each argument, and each component is relevant to the performance of the system. If the components' lifetimes are denoted by $T_{1}, \ldots, T_{n}$, then $T=\phi\left(T_{1}, T_{2}, \ldots, T_{n}\right)$ represents the lifetime of the system.

Let $T_{1}, T_{2}, \ldots, T_{n}$ denote the i.i.d. lifetime random variables with continuous distribution. Samaniego [12] introduced the signature of a coherent system $T=\phi\left(T_{1}, T_{2}\right.$, $\left.\ldots, T_{n}\right)$ as the vector $\mathbf{p}=\left(p_{1}, p_{2}, \ldots, p_{n}\right)$, where

$$
p_{i}=P\left\{\phi\left(T_{1}, T_{2}, \ldots, T_{n}\right)=T_{(i)}\right\}, \quad i=1, \ldots, n,
$$

where $T_{(i)}$ denotes the smallest $i$ th in $T_{1}, T_{2}, \ldots, T_{n}$, showing that

$$
\begin{aligned}
p_{i}= & \# \text { of orderings for which the } i \text { th failure causes } \\
& \text { system failure } \times(n !)^{-1} .
\end{aligned}
$$

A general formula for the reliability function of any coherent structure consisting of $n$ components can be given by using the concept of "signature" if the components are independent and identical. Samaniego [12] (see also [13]) showed that the reliability function of a coherent system $T=$ $\phi\left(T_{1}, T_{2}, \ldots, T_{n}\right)$ can be represented as

$$
P\{T>t\}=\sum_{i=1}^{n} p_{i} P\left\{T_{(i)}>t\right\} .
$$

Navarro et al. [14] (see also Navarro and Rychlik [15]) proved that the representation (26) also holds whenever $\left(T_{1}, T_{2}, \ldots, T_{n}\right)$ has an absolutely continuous exchangeable joint distribution. The following theorem provides the reliability function of any coherent structure consisting of $n$ components.

Theorem 7. Let $T_{i}$ denote the lifetime of the ith component whose strength is $Y_{i}(t), i=1,2, \ldots, n$, that is, $T_{i}=\inf \{s: s \geq$ $\left.0, X>Y_{i}(s)\right\}$. If $\phi$ denotes the structure function of the coherent system with lifetime $T$, that is, $T=\phi\left(T_{1}, T_{2}, \ldots, T_{n}\right)$ and $Y_{1}(t), Y_{2}(t), \ldots, Y_{n}(t)$ are i.i.d. with c.d.f. $G_{t}(x)=P\left\{Y_{i}(t) \leq\right.$ $x\}, i=1,2, \ldots, n$ then

$$
P\{T>t\}=\sum_{i=1}^{n} p_{i} P\left\{T_{(i)}>t\right\},
$$

where

$$
P\left\{T_{(i)}>t\right\}=\sum_{j=n-i+1}^{n} \sum_{m=0}^{n-j}(-1)^{m}\left(\begin{array}{c}
n \\
j
\end{array}\right)\left(\begin{array}{c}
n-j \\
m
\end{array}\right) E\left(\bar{G}_{t}(X)\right)^{j+m} .
$$

Proof. Under the assumption that $Y_{1}(t), Y_{2}(t), \ldots, Y_{n}(t)$ are i.i.d. the joint survival function of $T_{1}, T_{2}, \ldots, T_{n}$ is

$$
R\left(t_{1}, t_{2}, \ldots, t_{n}\right)=\int \prod_{i=1}^{n} \bar{G}_{t_{i}}(x) d F(x) .
$$

The function given by (33) is a mixture of independent $n$ variate d.f.'s with equal marginals; that is, the random vector $\left(T_{1}, T_{2}, \ldots, T_{n}\right)$ is positive dependent by mixture (PDM). PDM d.f.'s are exchangeable. (See, e.g., Shaked [16] for the concept of PDM and associated exchangeability). Since the representation (26) also holds for exchangeable lifetimes we get (27) with

$$
\begin{array}{r}
P\left\{T_{(i)}>t\right\}=\sum_{j=n-i+1}^{n}\left(\begin{array}{c}
n \\
j
\end{array}\right) P\left\{X<Y_{1}(t), \ldots, X<Y_{j}(t),\right. \\
\left.X \geq Y_{j+1}(t), \ldots, X \geq Y_{n}(t)\right\} .
\end{array}
$$

The usage of inclusion-exclusion principle for the probability inside the sum gives

$$
\begin{aligned}
P\left\{T_{(i)}>t\right\}= & \sum_{j=n-i+1}^{n}\left(\begin{array}{c}
n \\
j
\end{array}\right) \sum_{m=0}^{n-j}(-1)^{m}\left(\begin{array}{c}
n-j \\
m
\end{array}\right) \\
& \times P\left\{X<Y_{1}(t), \ldots, X<Y_{j+m}(t)\right\} .
\end{aligned}
$$

The proof is now completed by conditioning on $X$.

\section{Weibull Stress-Strength Model}

In this section we study the stress-strength reliability for the Weibull process which can be used to model the decreasing strength of a unit. Chiodo and Mazzanti [17] studied stressstrength reliability and its estimation for aged power system components subjected to voltage surges using Weibull process.

Let $Y(t)$ be a Weibull process whose one-dimensional distribution is

$$
G_{t}(x)=P\{Y(t) \leq x\}=1-\exp \left\{-\left(\frac{x}{\alpha(t)}\right)^{\beta}\right\}, \quad x>0,
$$


where the shape parameter $\beta$ is assumed to be time independent and the intensity function $\alpha(t)$ is decreasing in time with $\alpha(0)=\infty$. Similarly, assume that the stress random variable $X$ has a Weibull distribution with c.d.f.

$$
F(x)=1-\exp \left\{-\left(\frac{x}{\theta}\right)^{\beta}\right\}, \quad x>0, \theta, \beta>0 .
$$
to be

Under these assumptions the reliability function is found

$$
R(t)=\frac{1}{1+(\theta / \alpha(t))^{\beta}}, \quad t>0 .
$$

The following results can be obtained from Proposition 3.

Corollary 8. Let $T_{i}$ denote the lifetime of the system whose stress-strength pair is $\left(X_{i}, Y(t)\right), i=1,2$, where $X_{i}$ has a Weibull distribution with scale parameter $\theta_{i}$ and shape parameter $\beta$ and $Y(t)$ is a Weibull process whose distribution is given by (32). Then, since $g(x, t)=(\partial / \partial x) G_{t}(x)=$ $(\beta / \alpha(t))(x / \alpha(t))^{\beta-1} e^{-(x / \alpha(t))^{\beta}}$ is $R R_{2}$ in $(x, t)$, if $\theta_{1} \leq \theta_{2}$ then $T_{1} \stackrel{\mathrm{hr}}{\geq} T_{2}$.

Corollary 9. Under the same assumptions of Corollary 8, the function

$$
h(x, t)=\frac{\partial}{\partial t} G_{t}(x)=-\beta \frac{x^{\beta}}{\alpha^{\beta+1}(t)} \alpha^{\prime}(t) e^{-(x / \alpha(t))^{\beta}}
$$

is $R R_{2}$ in $(x, t)$. Indeed, because $\alpha(t)$ is decreasing, $\alpha^{\prime}(t)<0$, and hence $h(x, t) \geq 0$. For $x_{1}<x_{2}$ and $t_{1}<t_{2}$,

$$
h\left(x_{1}, t_{1}\right) h\left(x_{2}, t_{2}\right)-h\left(x_{1}, t_{2}\right) h\left(x_{2}, t_{1}\right) \leq 0
$$

which implies that $h(x, t)$ is $R R_{2}$ in $(x, t)$. Therefore, from Proposition 3, if $\theta_{1} \leq \theta_{2}$ then $T_{1} \stackrel{\operatorname{lr}}{\geq} T_{2}$.

Remark 10. If $\alpha(t)=1 / t$ then

$$
R(t)=\frac{1}{1+(\theta t)^{\beta}}, \quad t>0
$$

which is the survival function of the log-logistic distribution. That is, the lifetime of the system has log-logistic distribution with scale parameter $\lambda=1 / \theta$ and shape parameter $\beta$. If $\beta>1$ then the MTTF of the system is found to be

$$
E(T)=\frac{\pi / \theta \beta}{\sin (\pi / \beta)} .
$$

Theorem 11. Let $Y_{i}(t)$ be a Weibull process with intensity $\alpha_{i}(t)$ associated with ith component, $i=1, \ldots, n$. Assume that the common random stress $X$ has a Weibull distribution with c.d.f. given by (33). Then the joint survival function of $T_{1}, \ldots, T_{n}$ is

$$
R\left(t_{1}, \ldots, t_{n}\right)=\frac{1}{1+\left(\theta / \alpha_{1}\left(t_{1}\right)\right)^{\beta}+\cdots+\left(\theta / \alpha_{n}\left(t_{n}\right)\right)^{\beta}},
$$

and the survival copula associated with (39) belongs to the Clayton family and is given by

$$
C\left(u_{1}, \ldots, u_{n}\right)=\frac{1}{1 / u_{1}+\cdots+1 / u_{n}-n+1} .
$$

Proof. Using (19) one can write

$$
\begin{aligned}
R\left(t_{1}, \ldots, t_{n}\right) & \\
& =P\left\{X<Y_{1}\left(t_{1}\right), \ldots, X<Y_{n}\left(t_{n}\right)\right\} \\
& =\int_{0}^{\infty} e^{-\left(x / \alpha_{1}\left(t_{1}\right)\right)^{\beta}} \cdots e^{-\left(x / \alpha_{n}\left(t_{n}\right)\right)^{\beta}} \frac{\beta}{\theta}\left(\frac{x}{\theta}\right)^{\beta-1} e^{-(x / \theta)^{\beta}} d x \\
& =\frac{1}{1+\left(\theta / \alpha_{1}\left(t_{1}\right)\right)^{\beta}+\cdots+\left(\theta / \alpha_{n}\left(t_{n}\right)\right)^{\beta}} .
\end{aligned}
$$

By the definition of survival copula (see, e.g., Nelsen [18, page 32])

$$
\begin{aligned}
C & \left(u_{1}, \ldots, u_{n}\right) \\
& =R\left(R_{1}^{-1}\left(t_{1}\right), \ldots, R_{n}^{-1}\left(t_{n}\right)\right) \\
& =R\left(\alpha_{1}^{-1}\left(\theta\left(\frac{1}{u_{1}}-1\right)^{-1 / \beta}\right), \ldots, \alpha_{n}^{-1}\left(\theta\left(\frac{1}{u_{n}}-1\right)^{-1 / \beta}\right)\right) \\
& =\left(u_{1}^{-1}+\cdots+u_{n}^{-1}-n+1\right)^{-1}
\end{aligned}
$$

which is known to be a Clayton copula (see, e.g., Nelsen [18, page 152]). Thus the proof is completed.

Remark 12. For $\alpha_{i}(t)=1 / t, i=1, \ldots, n$, (39) becomes the survival function of the multivariate log-logistic distribution generated by the Clayton family of copulas.

Example 13. Consider the system consisting of $n$ components whose deteriorating strengths are modeled by a Weibull process with the common intensity function $\alpha(t)$. Suppose that these components are subjected to a common random stress $X$ which has c.d.f. given by (33). Then, the lifetimes of the components are exchangeable and we have

$$
E\left(\bar{G}_{t}(X)\right)^{j+m}=\frac{1}{1+(j+m)(\theta / \alpha(t))^{\beta}} .
$$

If, for example, a system has a 2-out-of-3 structure, that is, the system functions if and only if at least two components function, then since $\mathbf{p}=(0,1,0)$ using Theorem 7 , the reliability of the system is found to be

$$
P\left\{T_{(2)}>t\right\}=\frac{3}{1+2(\theta / \alpha(t))^{\beta}}-\frac{2}{1+3(\theta / \alpha(t))^{\beta}} .
$$

\section{Acknowledgment}

The author thanks the anonymous referee for his/her helpful comments and suggestions.

\section{References}

[1] R. A. Johnson, "Stress-strength models for reliability," in Handbook of Statistics, P. R. Krishnaiah and C. R. Rao, Eds., vol. 7, pp. 27-54, Elsevier, Amsterdam, North-Holland, 1988. 
[2] S. Kotz, Y. Lumelskii, and M. Pensky, The Stress-Strength Model and its Generalizations, World Scientific, River Edge, NJ, USA, 2003.

[3] G. A. Whitmore, "On the reliability of stochastic systems: a comment," Statistics \& Probability Letters, vol. 10, no. 1, pp. 65-67, 1990.

[4] N. Ebrahimi, "Two suggestions of how to define a stochastic stress-strength system," Statistics \& Probability Letters, vol. 3, no. 6, pp. 295-297, 1985.

[5] M. Shaked and J. G. Shanthikumar, Stochastic Orders, Springer Series in Statistics, Springer, New York, NY, USA, 2007.

[6] S. Karlin, Total Positivity. Vol. I, Stanford University Press, Stanford, Calif, USA, 1968.

[7] G. K. Bhattacharyya and R. A. Johnson, "Estimation of reliability in a multicomponent stress-strength model," Journal of the American Statistical Association, vol. 69, pp. 966-970, 1974.

[8] S. Chandra and D. B. Owen, "On estimating the reliability of a component subject to several different stresses (strengths)," Naval Research Logistics Quarterly, vol. 22, pp. 31-39, 1975.

[9] M. Pandey, M. B. Uddin, and J. Ferdous, "Reliability estimation of an s-out-of-k system with non-identical component strengths: the Weibull case," Reliability Engineering and System Safety, vol. 36, no. 2, pp. 109-116, 1992.

[10] S. Eryilmaz, "Consecutive k-out-of $n$ : $G$ system in stressstrength setup," Communications in Statistics. Simulation and Computation, vol. 37, no. 3-5, pp. 579-589, 2008.

[11] S. Eryilmaz, "Multivariate stress-strength reliability model and its evaluation for coherent structures," Journal of Multivariate Analysis, vol. 99, no. 9, pp. 1878-1887, 2008.

[12] F. Samaniego, "On closure of the IFR class under formation of coherent systems," IEEE Transactions on Reliability, vol. 34, no. 1, pp. 69-72, 1985.

[13] S. Kochar, H. Mukerjee, and F. J. Samaniego, "The "signature" of a coherent system and its application to comparisons among systems," Naval Research Logistics, vol. 46, no. 5, pp. 507-523, 1999.

[14] J. Navarro, J. M. Ruiz, and C. J. Sandoval, "A note on comparisons among coherent systems with dependent components using signatures," Statistics \& Probability Letters, vol. 72, no. 2, pp. 179-185, 2005.

[15] J. Navarro and T. Rychlik, "Reliability and expectation bounds for coherent systems with exchangeable components," Journal of Multivariate Analysis, vol. 98, no. 1, pp. 102-113, 2007.

[16] M. Shaked, "A concept of positive dependence for exchangeable random variables," The Annals of Statistics, vol. 5, no. 3, pp. 505-515, 1977.

[17] E. Chiodo and G. Mazzanti, "Bayesian reliability estimation based on a weibull stress-strength model for aged power system components subjected to voltage surges," IEEE Transactions on Dielectrics and Electrical Insulation, vol. 13, no. 1, pp. 146-159, 2006.

[18] R. B. Nelsen, An Introduction to Copulas, Springer Series in Statistics, Springer, New York, NY, USA, 2nd edition, 2006. 

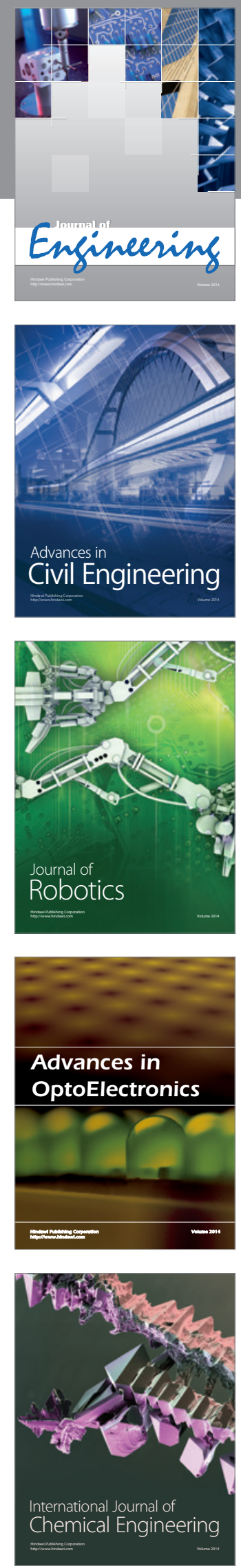

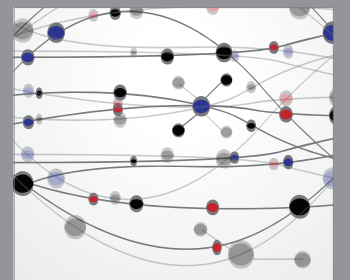

The Scientific World Journal
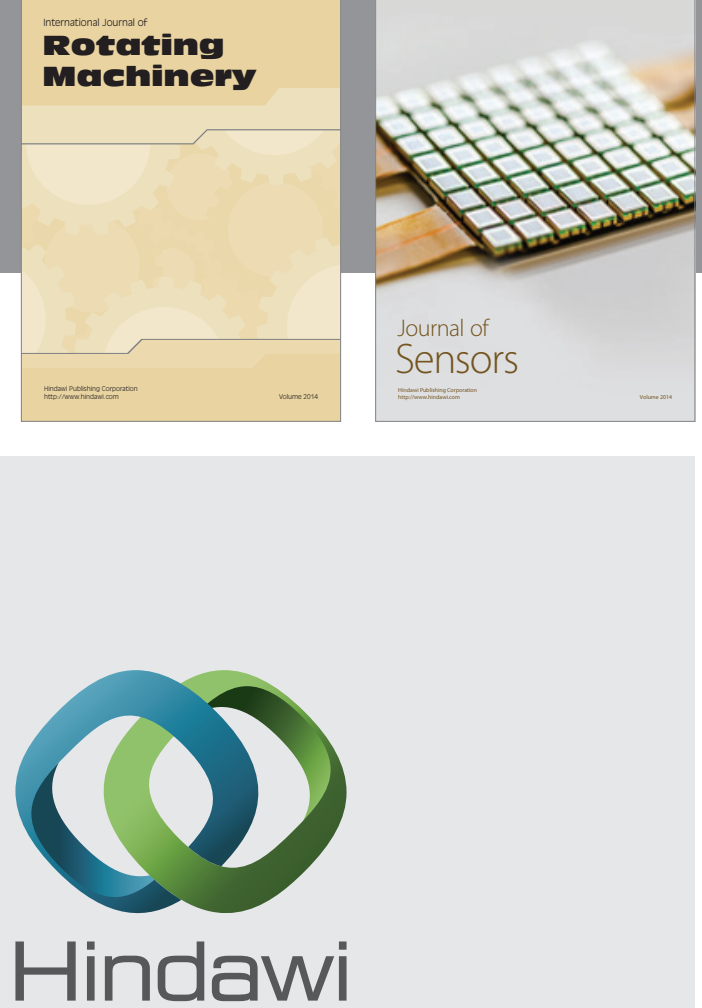

Submit your manuscripts at http://www.hindawi.com
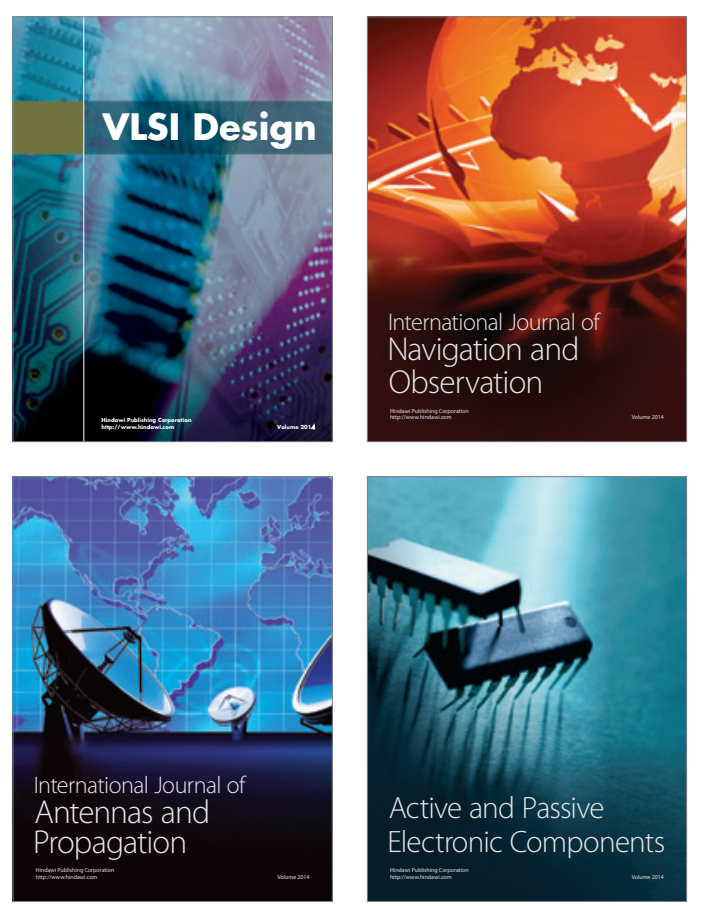
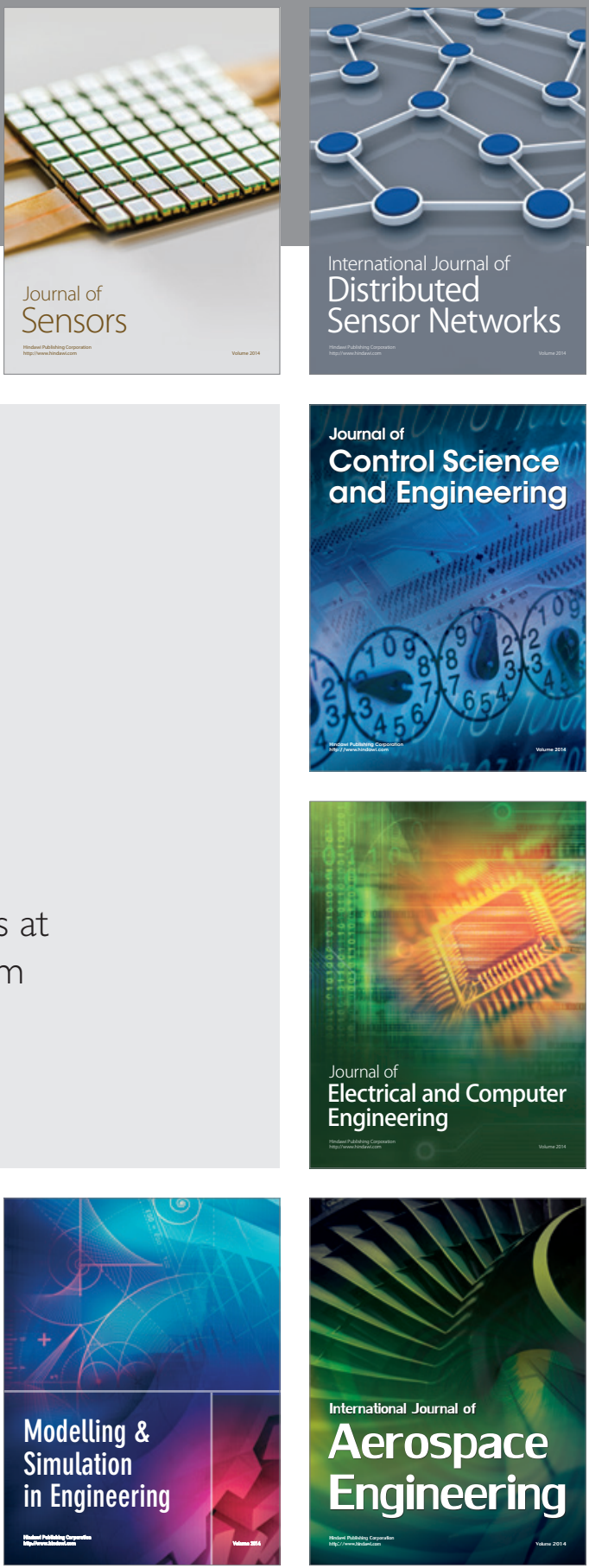

Journal of

Control Science

and Engineering
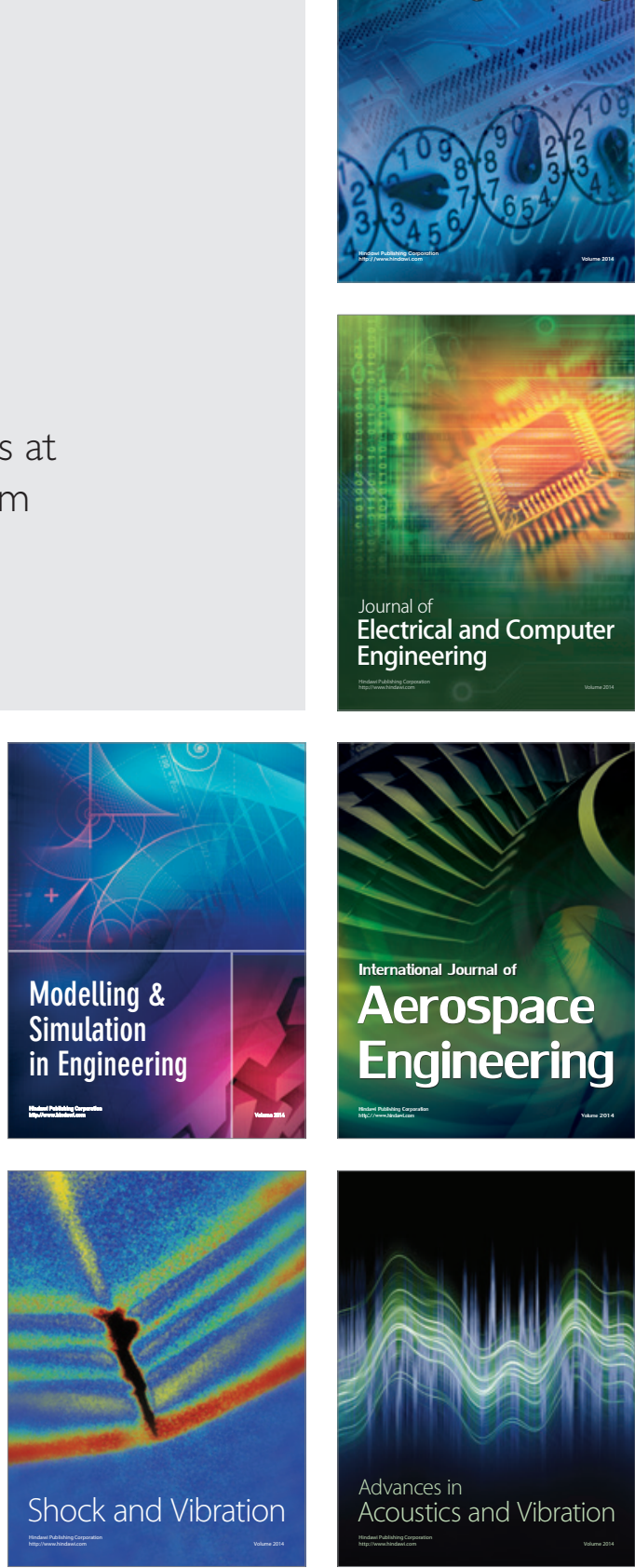